\title{
Sharp-edged vs. diffuse illusory circles: The effects of varying luminance
}

\author{
THEODORE E. PARKS and WILLIAM MARKS \\ University of California, Davis, California
}

\begin{abstract}
As had been found previously, one experiment demonstrated that reducing the luminance of a pattern that induced an abrupt-edged illusory figure increased mean ratings of that illusory effect. More importantly, the same result had not been found for a pattern that induced a diffuse illusory figure; in fact, a second experiment with such patterns produced a reliable tendency in the opposite direction. These results are at variance with the suggestion that an abrupt-edged illusory effect involves merely a minor variation in the boundary definition of a diffuse illusory lightness effect.
\end{abstract}

Schumann (1904) devised a pattern of black elements on a uniform white field that induces what is now called an illusory (or subjective) figure, which is to say, part of the uniform background appears to be lighter than the field around it. Specifically, the illusory figures seen in his and related patterns (e.g., Ehrenstein, 1941; Kanizsa, 1974) are characterized by relatively abrupt illusory lightness gradients marking their peripheries (see, e.g., the present Figure 1a). On the other hand, Kennedy $(1975,1978,1979)$ has constructed stimulus patterns that induce diffuseedged areas of illusory lightness (see, e.g., Figure 1b) and, moreover, has suggested that these effects are closely related to the earlier, abrupt-edged instances. Kennedy's suggestion is of the utmost theoretical importance, because it stands in opposition to the highly interesting possibility that relatively central, structural factors are involved in the production of the abrupt-edged brightness effect (see, e.g., Kanizsa, 1975).

In evaluating Kennedy's claim, Richardson (1979) compared the patterns of relative frequencies of reports of a bright, a dark, and a neither-bright-nordark illusory effect for a Kanizsa-type (i.e., abruptedged) illusory triangle and for a Kennedy-type (i.e., diffuse) illusory circle, when each stimulus type was viewed directly and when each was viewed peripherally. Richardson found a strong interaction of viewing style with type of illusion, suggesting the influence of fundamentally different mechanisms in the pro-

The authors would like to thank two anonymous reviewers for their many helpful suggestions and Ellen Lucas, Alan Templeton, Greg Millard, and especially Don Rasmussen for their assistance in collecting this data and for many helpful suggestions regarding the conduct and interpretation of this work. This research was supported by Grant D-262 from the University of California, Davis. Reprint requests should be addressed to the first author at the Department of Psychology, University of California, Davis, Davis, California 95616. duction of abrupt and of diffuse illusory lightness effects. However, recent work based on Richardson's technique has failed to reveal differences between diffuse and abrupt-edged illusory figures when the particular inducing patterns that were employed (and the illusory figures that resulted) were of similar configuration (Parks, 1982). Of course, this does not imply that other, more sensitive, techniques might not reveal differences even under those circumstances.

As one possibility, it is known that the level of illumination incident upon a pattern that normally yields an abrupt illusory figure is critical to its effectiveness. For example, Ronchi and Mori (1959) found that illusory figures that were readily apparent at moderate levels of illumination failed to occur when illumination was either increased or decreased beyond certain limits. Going further, Dumais and Bradley (1976) obtained reports of variation in the "strength or salience" of abrupt-edged illusory figures within that range and found clear evidence that reducing the level of illumination improved the effect. The purpose of the present research was to ascertain whether the same result would occur for a diffuse example. If not, of course, the important claim of a close kinship between diffuse and abrupt illusory figures would, once again, be cast in doubt.

\section{EXPERIMENT 1}

\section{Method}

Subjects and Materials. A total of $\mathbf{4 0}$ naive volunteers were individually shown copies of Figures $1 \mathrm{a}$ and $1 \mathrm{~b}$ in an Iconix 6137 tachistoscope. Each pattern was $11 \mathrm{~cm}$ in height and was presented at a distance of $86 \mathrm{~cm}$. As has been noted, Figure 1a is capable of inducing a sharp-edged illusory circle, whereas Figure ib tends to induce a diffuse one. Since the reflectance of all such materials was constant, changes in the level of illumination will hereafter be referred to as changes in luminance.

Procedure. Each subject first received general instructions that included presentation of each of the two stimulus patterns (but no specific suggestion as to their relative effectiveness) and that 

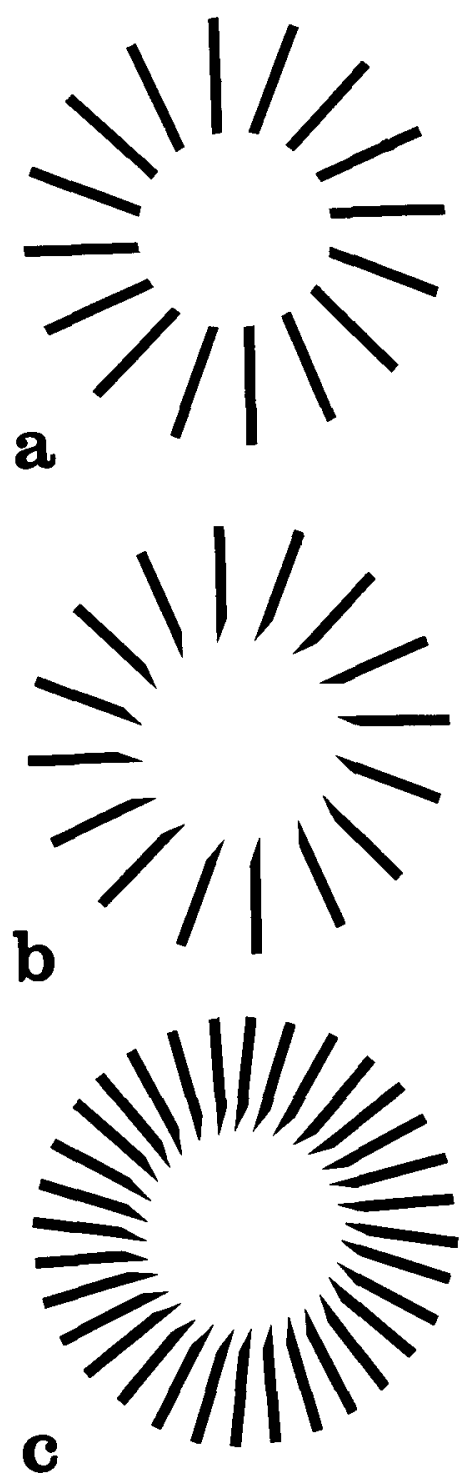

Figure 1. The stimulus patterns employed in Experiment 1 (a and b) and Experiment 2 (b and c). Pattern a induces an abruptedged illusory circle, whereas patterns $b$ and $c$ induce diffuse circles.

indicated that they would be shown patterns "like the ones you just saw" under a "variety of conditions." More specifically, they were told that each trial would consist of the presentation of a pattern accompanied by a capital $\mathbf{A}$ in the lower right-hand corner of the viewing screen (i.e., outside the circle of radiating lines), followed by an unmarked pattern, then by the marked pattern again, and, finally, by a last presentation of the unmarked pattern. Each presentation of either pattern was $2.8 \mathrm{sec}$ in duration and was separated from the next presentation by a blank field $.2 \mathrm{sec}$ in duration.

Except for the letter that marked one of them, the two patterns presented on any trial were always identical (either two copies of Figure la or two of Figure 1b). However, the luminance of the marked pattern was always $25 \mathrm{~cd} / \mathrm{m}^{2}$, whereas that of the unmarked pattern varied among $1.8,2.6,3.6,7.2$, and $32.0 \mathrm{~cd} / \mathrm{m}^{2}$. The luminance of the interstimulus field (which also served as an intertrial field) was set at $18 \mathrm{~cd} / \mathrm{m}^{2}$. Since the experimenter waited for (and recorded) the subject's response before he initiated the following trial, the intertrial interval varied, but was on the order of several seconds.

The subjects were asked to evaluate the salience of the illusion produced by the unmarked stimulus on each trial relative to that for the marked example. Specifically, they were told to notice "how real it seems, how powerful it is, how much it seems to fool you." They were further told to assign a value of " 50 " to the salience of the marked (the standard) example and to rate the effect produced by the unmarked (the comparison) pattern on a scale of " 0 to 100 " relative to the standard effect, "where ' 0 ' means no illusion at all and ' 100 ' means it is much stronger or more real to you." It should be further noted that the subjects were told that luminance would not always be the same and were told not to report any changes in overall luminance that they noticed.

Design. Each subject participated in 60 experimental trials following a series of five practice trials that were identical to that subject's first five experimental trials except that no data were recorded. The first 15 experimental trials involved one of the two pattern types (Figure 1a or 1b), followed by two blocks of 15 trials each of the other type, and finally by 15 of the first. Within any 15-trial blocks, there were 3 trials at each illumination level of the comparison stimulus. Specifically, the first 5 trials included 1 trial at each level of luminance (the order for a given subject consisting of one row of a $5 \times 5$ Latin square, all rows being employed equally across subjects), and this sequence was repeated twice more to yield a 15-trial block. One group, consisting of half of the subjects, received Figure la during their first block, and the other group viewed Figure $1 \mathrm{~b}$.

Since all data for any subject within a given combination of luminance and pattern were pooled, the design is three-factor, with groups as a between variable and luminance and pattern as within-subject variables.

After the data had been collected, each subject was given an informal interview in order to assess his or her understanding of the instructions. Specifically, he or she was required to finish the two sentences "During the experiment, I used large (small) numbers when __ One subject, who reported using smaller numbers "when the circle appeared much brighter than the background" (i.e., the inverse of the instructions that were given), was rejected and replaced.

\section{Results}

As can be seen in Figure 2, increasing luminance generally decreased the rated salience of the abruptcontoured illusory circles from a mean rating of $\mathbf{6 2 . 9}$ for the dimmest setting to a mean rating of 50.5 for the brightest. On the other hand, results for the diffuseedged case were, at best, much less impressive, the extreme mean ratings obtained being 52.4 and $\mathbf{5 0 . 5}$. Thus, not only was the main effect of luminance reliable $[F(4,152)=5.92, p<.01]$, but so were that for stimulus type $[F(1,38)=14.18, p<.01]$ and, most importantly, that for the interaction of luminance with stimulus type $[F(4,152)=22.70, p<.01]$. A subsequent analysis revealed that the linear component of the results obtained for the abrupt effect was highly reliable $[F(1,152)=77.13, p<.01]$ and accounts for $94 \%$ of the linear component of the interaction of luminance with stimulus type.

\section{Discussion}

As expected, at least within the middle range of the set of values employed, varying the luminance had 


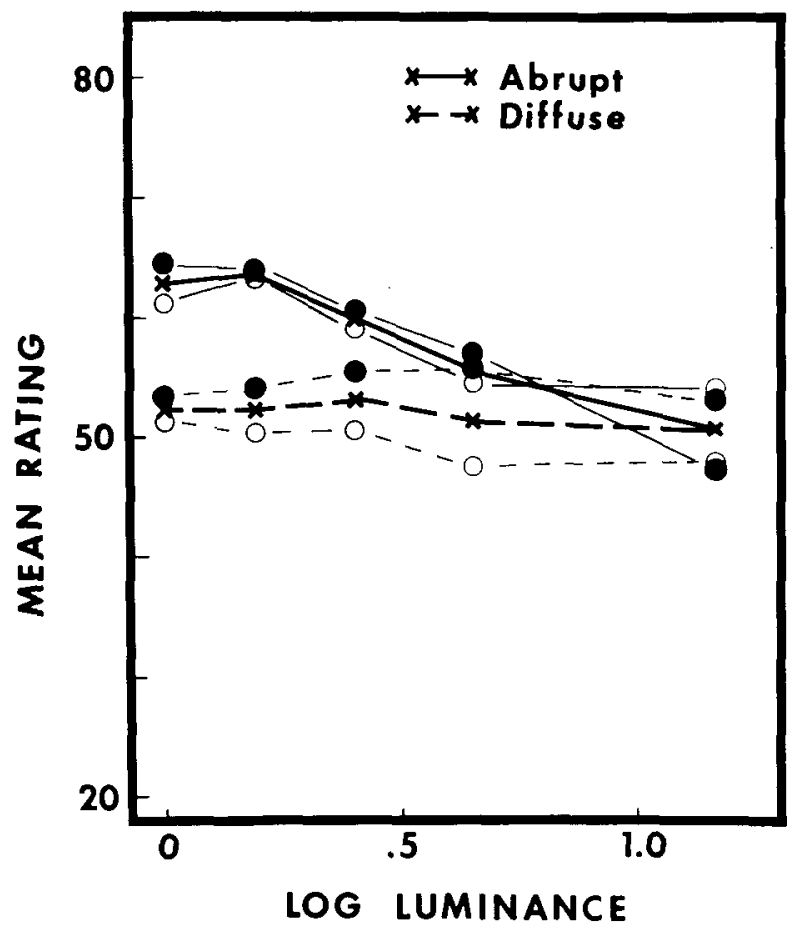

Figure 2. The effect of varying illumination on mean ratings of illusory contours that are abrupt (solid lines) and diffuse (broken lines) when both types are seen by all subjects (Experiment 1). The data for the group that were shown the abrupt example during their first block of trials (filled circles) are also shown separately from those for the group that first saw the diffuse effect (open circles).

a powerful effect on the type of illusion introduced by Schumann (1904) and exemplified here by Figure 1a. These results support not only the findings of Dumais and Bradley (1976) in a general way, but also tend to agree in some specifics with the suggestion of Ronchi and Mori (1959) that there are extreme values in either direction beyond which further decreases in luminance will produce no better effect (and, in fact, will tend to weaken it) and, at the opposite extreme, beyond which further increases in luminance will produce no poorer effect (since it is already poor). Specifically, mean ratings obtained for the present abrupt-edged instance at the two lowest levels were quite similar (62.9 for lowest and 63.7 for the next lowest), and the highest level yielded a mean rating close to that of the standard (50.5), despite its being brighter.

On the other hand, the point of major interest is the finding of little, if any, tendency toward an effect of luminance in the overall results obtained with Figure $1 \mathrm{~b}$, a finding that seriously threatens the contention that the diffuse effect seen there is closely related to the abrupt effect of Figure 1a.

However, a potentially powerful impediment to that conclusion must not be overlooked, an impediment that stems from the fact that the present in- structions were necessarily phrased in very general terms so as to be applicable to both diffuse and abrupt effects. To produce that degree of generality, the subjects were asked to rate "the illusion" without ever being told specifically what the illusion was. As a result, the subjects were left to discover for themselves that, for example, Figure $1 \mathrm{a}$ is capable of yielding an abrupt illusory contour. More importantly, they were free to decide (were, in fact, forced to decide) what specific criterion to apply in each case and whether to apply the same criterion to both types of effect. The very real danger is that once these subjects discovered the abrupt edges that were present on some trials, the strength of such edges might have been adopted as the basis for rating all instances. If so, of course, there would be no reason to expect variation among various diffuse examples, since they would necessarily all be equal (i.e., equally poor) in this regard. In other words, reducing luminance might, in fact, have increased the magnitude of the apparent decrement in lightness between the inside and the surround of the diffuse circle, but such an increase went unreported simply because its form was never abrupt (never steep enough), regardless of its total magnitude.

In addition to such logical considerations, there were empirical reasons to suggest that it might be propitious to obtain ratings of the diffuse effect from subjects who are not also required to observe (and rate) sharp-edged instances. That is, the results shown in Figure 2 reveal a slight, but noticeable, tendency for the group of subjects who observed diffuse examples in their first block of trials to rate dim diffuse effects as being more salient than well-lit ones. In fact, the interaction of luminance $\times$ stimulus type $X$ group was small but reliable: $F(4,152)=6.17$, $p<.01$. Although some of that interaction is undoubtedly due to differences in the ratings given to the abrupt instances, especially at extreme values, part of that interaction might also be the result of luminance changes producing an effect in the diffuse case that was parallel to (but weaker than) the effect produced in the abrupt illusion. That is, it is possible that such an effect was detected and reported by the group that saw the diffuse examples first, but was so unimpressive as to go unreported by those subjects who saw the (more impressive) changes in the abrupt examples first.

What was needed, then, was a reevaluation of the diffuse instances by subjects who were shown only that class of illusory effect and with instructions tailored to it.

\section{EXPERIMENT 2}

\section{Method}

Subjects and Materials. An additional group of $\mathbf{4 0}$ naive volunteers was shown Figure $1 \mathrm{~b}$ under the same physical conditions that 
prevailed in Experiment 1. In addition, a second pattern was sometimes used as the comparison stimulus (Figure 1c). This pattern, which also induces a diffuse illusory circle, but typically one of somewhat greater strength, was included because it was feared that illumination changes might, indeed, not affect the strength of the diffuse illusion. If that were the case, an experimental session consisting only of ratings of Figure $1 \mathrm{~b}$ would be exceedingly monotonous. In addition, inclusion of this new pattern provided a means of determining whether differential effects of varying luminance were due simply to differences in the power of the examples tested.

Procedure. The procedure duplicated that of Experiment 1, except that subjects were not shown an example of an abruptedged illusion during the instruction (or, of course, at any other time) and except that the wording of certain critical parts of the instructions, themselves, was different. Specifically, whereas the first instructions had referred to the strength "of the illusion" without further elaboration, these new instructions went on to include "In other words, how much does the center seem to glow compared to everything around it?" Most importantly, the subjects were again told not to simply report changes in the overall level of illumination.

Design. The design of this experiment duplicated that of Experiment 1 , except that a marked version of Figure $1 \mathbf{b}$ always served as the standard and, rather than biocks of trials involving Figure 1a or $\mathbf{l b}$ as the comparison pattern, each block employed either Figure $1 \mathrm{~b}$ or $1 \mathrm{c}$. Furthermore, blocks of 15 trials each were alternated to avoid a potentially monotonous block of 30 identical trials in the middle of each experimental session. The concluding interviews revealed insufficient grounds for replacing any subject.

\section{Results}

Even when presented exclusively, the diffuse illusory effect induced by Figure $1 \mathrm{~b}$ was not affected by changes in luminance in a way that paralleled the results that have been obtained for abrupt-edged instances. On the contrary, as can be seen in Figure 3, it was affected in the opposite direction. For example, whereas the effect seen in Figure $1 \mathrm{~b}$ was rated as quite similar to the standard (the same pattern) when its luminance was high, its rated effectiveness noticeably decreased (rather than increased) as luminance was reduced (extreme mean ratings being 46.8 and 34.4). Similarly, while Figure 1c received a higher mean rating than the standard when both were bright (the mean being 76.9 ), that advantage gradually eroded as the luminance of Figure $1 \mathrm{c}$ was reduced (the mean becoming 63.7 , a value that was coincidentally similar to the highest mean ratings of Experiment 1). As a result, reliable effects of luminance $[F(4,152)=$ $20.26, p<.01]$ and of stimulus pattern $[F(1,38)=$ $110.16, \mathrm{p}<.01]$ were obtained, and the interaction of those variables was small and unreliable $[F(4,152)$ $<1.0]$.

It should be noted that individual group data are not included in Figure 3 because the results for the two groups were exceedingly similar. For example, the largest difference between group means occurred for the dimmest luminance of Figure $1 \mathrm{~b}$, the mean ratings being $\mathbf{3 2 . 6}$ for the group that saw that figure on their first block of trials and 36.1 for the other group. As a result, the $\mathrm{F}$ ratios for the main effect of

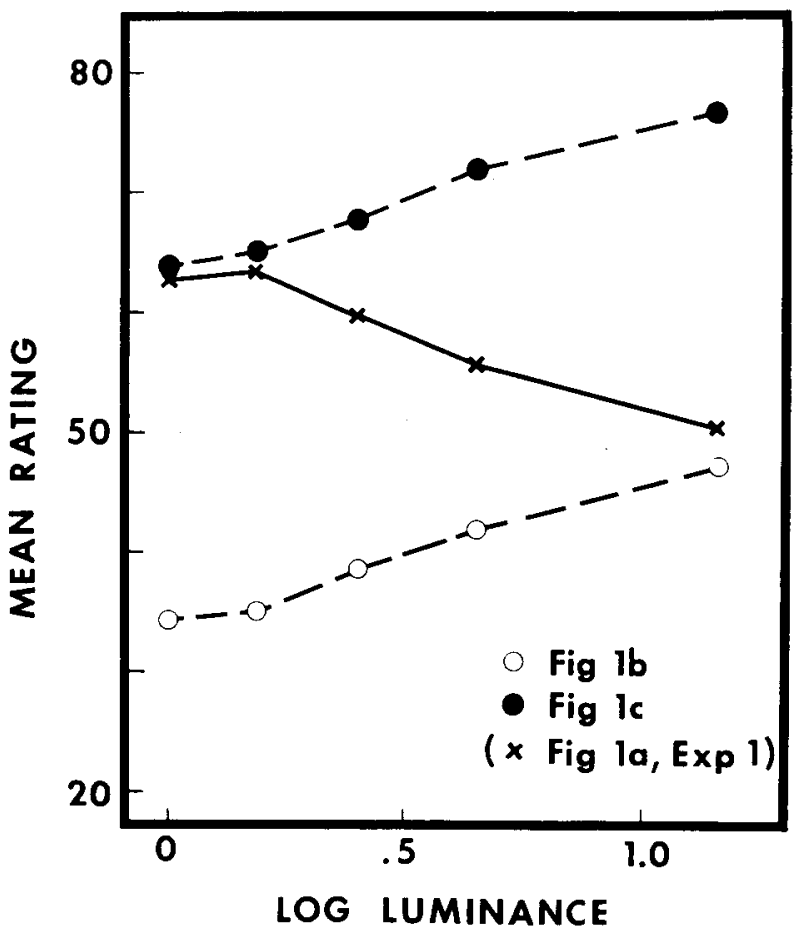

Figure 3. The effect of varying illumination on mean ratings of the diffuse illusions induced by two stimulus patterns for subjects who were only shown diffuse instances (Experiment 2). For ease of comparison, the overall results obtained for the abrupt-edged illusory figure of Experiment 1 are also reproduced here.

groups and all of its interactions were clearly unreliable (the largest being 1.02).

\section{Discussion}

Apparently, the relative lightness of the diffuse illusory effects induced by Figures $1 \mathrm{~b}$ and $1 \mathrm{c}$ depends upon luminance, but in a manner that is opposite to the results obtained in the first experiment for Figure la and by Dumais and Bradley (1976) for another abrupt-edged illusory figure. Some caution is indicated in interpreting these opposing results since, of course, the subjects in Experiment 2 were explicitly asked to judge illusory lightness, whereas those of the first were asked, more generally, to judge salience, which they might have understood to mean lightness or to mean clarity or depth or some combination of two or more of these qualities. This problem is, however, not a serious one in light of previous evidence that these qualities covary across manipulations of inducing stimuli. For example, Jory and Day (1979) found that changes in the mean rating of the lightness effect that were produced by degrading (in various ways) dark-on-light patterns very similar to the present abrupt example were highly correlated with corresponding changes in the mean rating of clarity brought about by the same degradations in a separate group 
of subjects (compare, e.g., Jory \& Day's Conditions $\mathrm{E}$ and $B$ or $G$ and $C$ ).

Consequently, the present interaction of luminance with illusion type must be taken as casting some doubt upon the suggestion that abrupt and diffuse illusory figures share underlying mechanisms to any great extent. Instead, it may be that abrupt illusory contours depend to a large extent upon organizational factors (as per, for example, the suggestions of Kanizsa, 1975, Parks, Rock, \& Anson, in press, and Rock \& Anson, 1979), which are relatively absent in diffuse cases. On the other hand, diffuse illusory gradients may depend upon certain specific details (such as sharp points) of the inducing pattern that need not be dominant elements of (or even present in) patterns that induce abrupt gradients. Furthermore, the specific consequences of varying the level of illumination incident upon the present diffuse patterns suggests a major contribution by some form of simultaneous contrast (including, perhaps, a very special one; see, e.g., Frisby \& Clatworthy, 1975), which, judging by the opposite effects obtained, must play a relatively insignificant role in the production of abrupt illusoryfigure gradients.

\section{REFERENCES}

Dumais, S. T., \& Bradley, D. R. The effects of illumination level and retinal size on the apparent strength of subjective contours. Perception \& Psychophysics, 1976, 19, 339-345.

Ehrenstein, W. Über Abwandlungen der L. Hermannschen Helligkeitserscheinung. Zeitschrift fur Psychologie, 1941, 150, 83-91.
Frisby, J. P., \& Clatworthy, J. L. Illusory contours: Curious cases of simultaneous brightness contrast? Perception, 1975, 4, 349-357.

JoRY, M. K., \& DAY, R. H. The relationship between brightness contrast and illusory contours. Perception, 1979, 8, 3-9.

Kanizsa, G. Contours without gradients or cognitive contours? Italian Journal of Psychology, 1974, 1, 93-113.

Kanizsa, G. Some new demonstrations of structural factors in brightness contrast. In S. Ertel, L. Kemmler, \& M. Stadler (Eds.), Gestaltheorie in der moderne Psychologie. Darmstadt: Steinkopff, 1975.

Kennedy, J. M. Depth at an edge, coplanarity, slant depth, change in direction and change in brightness in the production of subjective contours. Italian Journal of Psychology, 1975, 2, 107-123.

Kennedy, J. M. Illusory contours not due to completion. Perception, 1978, 7, 187-189.

KENNEDY, J. M. Subjective contours, contrast, and assimilation. In C. F. Nodine and D. F. Fisher (Eds.), Perception and Pictorial Representation. New York: Praeger, 1979.

PARKs, T. E. Brightness effects in diffuse and sharp illusory figures of similar configuration. Perception, 1982, 11, 107-110.

Parks, T. E., Rock, I., \& Anson, R. Illusory contour lightness: A neglected possibility. Perception, in press.

Richardson, B. L. The nonequivalence of abrupt and diffuse illusory contours. Perception, 1979, 8, 589-593.

Rock, I., \& Anson, R. Illusory contours as the solution to a problem. Perception, 1979, 8, 665-681.

Ronchi, L., \& MORI, G. F. On the factors which affect the contrast enhancement in a figure with "quasi perceptive contours" and a practical application of such a figure. Fondazione Giorgio Ronchi Atti, 1959, 14, 495-508.

Schumann, F. Einige Beobachtungen über die Zusammenfassung von Gesichtseindrucken zu Einheiten. Psychologische Studien, $1904,1,1-32$.

(Manuscript received August 2, 1982; revision accepted for publication November 30,1982 .) 